the onset of chromosome instability? How might the status of PinX 1 in tumors guide therapy? The authors suggest that telomerase inhibitors may be useful for treating cancers lacking PinX1 function. This is an intriguing idea, provided the benefit to cancer cells of PinX1 loss persists in established tumors (e.g., via promoting telomere lengthening); however, if the key benefit occurs early, via enhanced genome instability, PinX1 status may not affect the sensitivity of mature tumors to telomerase inhibition. Fortunately, existing tools can be combined to address whether cancer in PinX1 $1^{+/-}$mice or the growth of PinX1-deficient tumor cell lines is particularly susceptible to telomerase inhibition, and so answers should soon be forthcoming.

\section{Acknowledgments}

The author thanks P. Lieberman, Y. Tzfati, R. Greenberg, and members of the Johnson lab for helpful discussions. This work was supported by NIH grant AG021521.

Address correspondence to: F. Brad Johnson, Department of Pathology and Laboratory Medicine, University of Pennsylvania
School of Medicine, 422 Curie Boulevard, Philadelphia, Pennsylvania 19104, USA. Phone: 215.573.5057; Fax: 215.573.6317; E-mail: johnsonb@mail.med.upenn.edu.

1. Palm W, de Lange T. How shelterin protects mammalian telomeres. Annu Rev Genet. 2008;42:301-334.

2. Kovalenko OA, et al. A mutant telomerase defective in nuclear-cytoplasmic shuttling fails to immortalize cells and is associated with mitochondrial dysfunction. Aging Cell. 2010;9(2):203-219.

3. Artandi SE, DePinho RA. Telomeres and telomerase in cancer. Carcinogenesis. 2010;31(1):9-18.

4. Park JI, et al. Telomerase modulates Wnt signalling by association with target gene chromatin. Nature. 2009; 460(7251):66-72.

5. Flores I, Benetti R, Blasco MA. Telomerase regulation and stem cell behaviour. Curr Opin Cell Biol. 2006;18(3):254-260.

6. Soohoo CY, Shi R, Lee TH, Huang P, Lu KP, Zhou $X Z$. Telomerase inhibitor PinX1 provides a link between TRF1 and telomerase to prevent telomere elongation.J Biol Chem. 2011;286(5):3894-3906.

7. Davalos AR, Coppe JP, Campisi J, Desprez PY. Senescent cells as a source of inflammatory factors for tumor progression. Cancer Metastasis Rev. 2010;29(2):273-283.

8. Thompson SL, Compton DA. Chromosomes and cancer cells [published online ahead of print December 29, 2010]. Chromosome Res. doi:10.1007/ s10577-010-9179-y.

9. Artandi SE, et al. Telomere dysfunction promotes non-reciprocal translocations and epithelial cancers in mice. Nature. 2000;406(6796):641-645.

10. Emi M, et al. Frequent loss of heterozygosity for loci on chromosome $8 \mathrm{p}$ in hepatocellular carcinoma, colorectal cancer, and lung cancer. Cancer Res. 1992;52(19):5368-5372.

11. Zhou XZ, et al. The telomerase inhibitor PinX1 is a major haploinsufficient tumor suppressor essential for chromosome stability in mice. J Clin Invest. 2011;121(4):1266-1282.

12. Yuan $\mathrm{K}$, et al. PinX1 is a novel microtubule-binding protein essential for accurate chromosome segregation. J Biol Chem. 2009;284(34):23072-23082.

13. Yoo JE, Oh BK, Park YN. Human PinX1 mediates TRF1 accumulation in nucleolus and enhances TRF1 binding to telomeres. J Mol Biol. 2009; 388(5):928-940.

14. Lin J, Blackburn EH. Nucleolar protein PinX1p regulates telomerase by sequestering its protein catalytic subunit in an inactive complex lacking telomerase RNA. Genes Dev. 2004;18(4):387-396.

15. Morales CP, et al. Absence of cancer-associated changes in human fibroblasts immortalized with telomerase. Nat Genet. 1999;21(1):115-118.

16. Li B, Lustig AJ. A novel mechanism for telomere size control in Saccharomyces cerevisiae. Genes Dev. 1996;10(11):1310-1326.

17. Gonzalez-Suarez E, Flores JM, Blasco MA. Cooperation between $\mathrm{p} 53$ mutation and high telomerase transgenic expression in spontaneous cancer development. Mol Cell Biol. 2002;22(20):7291-7301.

18. Gao Q, et al. Telomerase-dependent and -independent chromosome healing in mouse embryonic stem cells. DNA Repair (Amst). 2008;7(8):1233-1249.

19. Boule JB, Zakian VA. Roles of Pif1-like helicases in the maintenance of genomic stability. Nucleic Acids Res. 2006;34(15):4147-4153

20. Sfeir A, et al. Mammalian telomeres resemble fragile sites and require TRF1 for efficient replication. Cell. 2009;138(1):90-103.

\title{
The adaptive stroma joining the antiangiogenic resistance front
}

\author{
Oriol Casanovas
}

Tumor Angiogenesis Group, Translational Research Laboratory, Catalan Institute of Oncology — IDIBELL, L'Hospitalet de Llobregat, Spain.

\begin{abstract}
Resistance to antiangiogenic therapies in cancer involves both tumor cells and stromal components, but their relative contributions differ in each cancer subtype. In this issue of the JCI, Cascone et al. describe a stromal adaptation to antiangiogenic therapy in non-small cell lung carcinoma (NSCLC) models that include EGFR-driven vascular remodeling promoting resistance to VEGF inhibition. Their results suggest that the added benefit of dual VEGF/R and EGFR targeting in these models could be clinically relevant to fight resistance in NSCLC patients.
\end{abstract}

The growth of a tumor depends on vascular remodeling to ensure a continuous supply of nutrients and oxygen, and blockade of the formation of new blood vessels with antiangiogenic drugs is currently used to treat certain types of cancer. For its central role in promoting angiogenesis, VEGF is

Conflict of interest: The author has declared that no conflict of interest exists.

Citation for this article: JClin Invest. doi:10.1172/ JCI46430. the main target of the currently approved antiangiogenic drugs. Nevertheless, clinical results demonstrate only moderate gains in time to progression and scarce benefits in overall survival, despite long-term treatment (1). Why are there such modest and short-lasting benefits of antiangiogenic therapies in the clinic? The initial hypothesis was that antiangiogenic therapy would not induce resistance (i.e., "resistant to resistance") because it targeted endothelial cells instead of the tumor cell itself (2). Never- theless, clinical and experimental evidence has been mounting that resistance to antiangiogenic therapy does indeed occur (3).

Among the tumor responses to therapy, it is critical to distinguish between refractoriness, sometimes called intrinsic resistance (4), and acquired resistance. Tumors have long been shown to have remarkable plasticity and adaptability to classical chemotherapy and radiation, and this plasticity contributes to evasion from antiangiogenic therapy (5-7). However, the specific mechanisms of acquired resistance to antiangiogenic therapies are unique, and may reverse after antiangiogenic therapy has been stopped (M. Pàez-Ribes and O. Casanovas, unpublished observations). This suggests that these forms of resistance may reflect adaptations to therapy rather than the mutations or gene amplifications that characterize acquired resistance to other thera- 


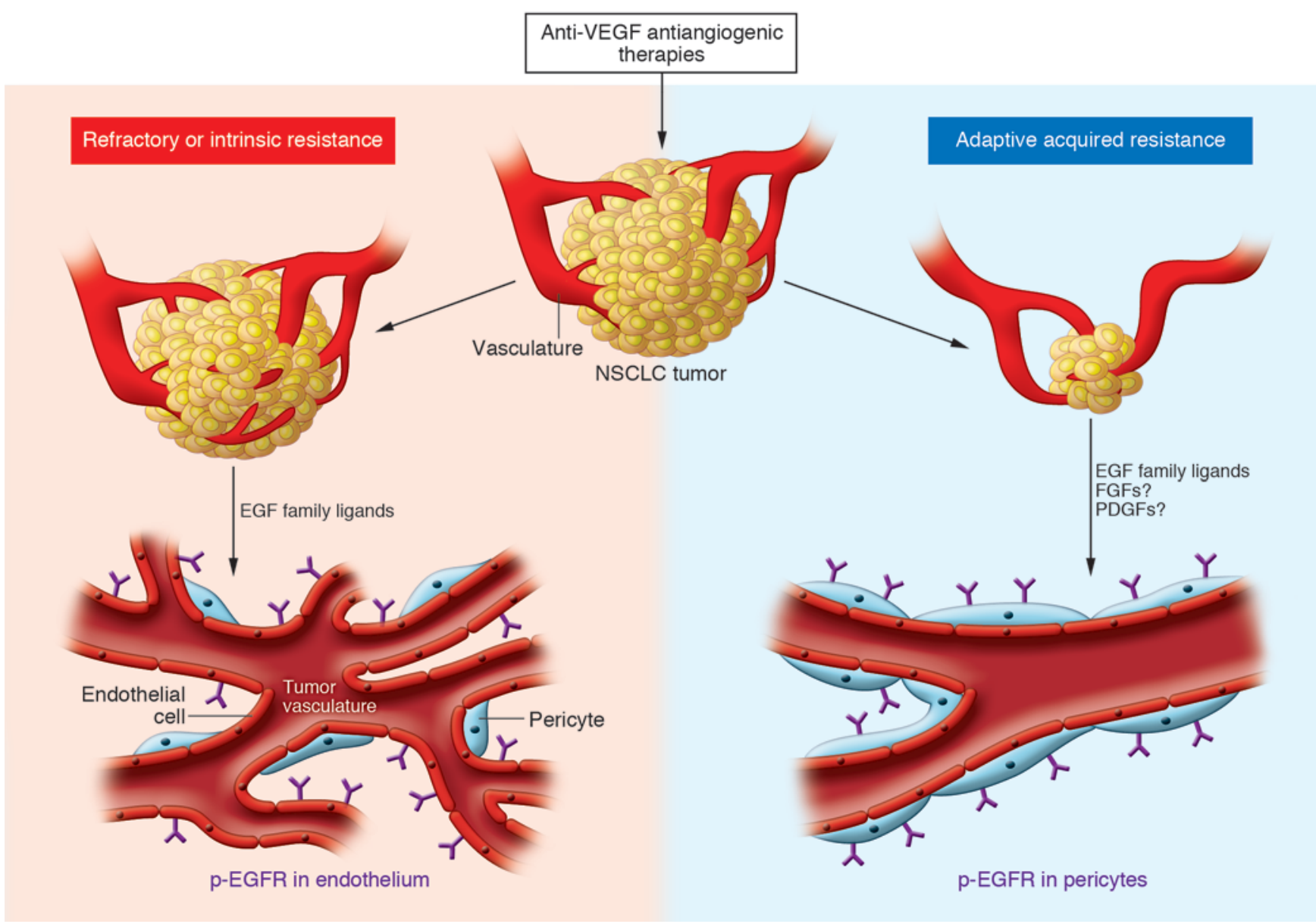

Figure 1

Stromal adaptation to induce resistance to antiangiogenic therapies. Multiple mechanisms of resistance to antiangiogenic therapies arise depending on the tumor type and microenvironment, and the different stromal components and functions are key for the full resistance phenotype. Cascone et al. (13) tackle the question of resistance to anti-VEGF therapy in NSCLC tumors by using a relatively refractory xenograft model (left) and two acquired resistance models (right). In the partially refractory model, anti-VEGF therapy does not alter tumor growth, but induces disorganized sprouting revascularization that leads to more tortuous vasculature with lower pericyte coverage, concomitant with endothelial upregulation of $p$-EGFR in treated tumors. In the case of acquired resistance, tumors initially respond to anti-VEGF therapy, but rapidly acquire resistance with efficient revascularization following a pattern of pericyte-covered normalized vasculature with increased activated EGFR on perivascular cells. Differences in the repertoire of proangiogenic growth factors expressed in each of these cases drive the mechanisms of stromal resistance to antiangiogenic therapies.

peutic strategies. Indeed, clinical evidence of this reversibility has been described in metastatic renal cell carcinoma repeatedly treated with VEGFR small-molecule inhibitors (3). The adaptive nature of the resistance to antiangiogenic therapy suggests that this process might be easily targeted in order to improve the clinical outcome.

\section{Stromal resistance to antiangiogenics}

In addition to the tumor cells themselves, many different host factors and stromal components have been described to play an important role in resistance to angiogenesis inhibitors $(8,9)$. For instance, several mechanisms of refractoriness to antiangio- genic therapies implicate stromal cells in conferring intrinsic resistance to anti-VEGF monoclonal antibody, which ultimately ensures the continuous formation of tumor blood vessels even when signaling through VEGFR is inhibited (10-12). In contrast, there are few published demonstrations of stromal components playing a key role in acquired resistance to antiangiogenic therapies. In this issue, Cascone et al. tackle the question of stromal adaptation and resistance to antiangiogenic therapies from a global gene expression approach that discriminates host from tumor cells (13). Their findings suggest that in xenograft models of non-small cell lung carcinoma (NSCLC), resistance to VEGF inhibition occurs with adaptive changes in the tumor stroma, including EGFR-driven vascular remodeling that allows for tumor regrowth.

First, Cascone et al. describe a relatively refractory xenograft model (A549 cells) in which anti-VEGF therapy, while not altering tumor growth, induces disorganized sprouting revascularization that leads to more tortuous vasculature with lower pericyte coverage (Figure 1 and ref. 13). In this case, the endothelial upregulation of p-EGFR in treated tumors suggests that one of the possible mechanisms of intrinsic resistance in this model could be associated with a switch to EGFR-driven endothelial proliferation and angiogenesis in the face of antiVEGF therapy. 
In two cases of acquired resistance (subcutaneous H1975 cells and orthotopic H441 cells), revascularization occurred in the resistance phase with a pattern of pericyte-covered normalized vasculature and increased activated EGFR on perivascular cells (Figure 1). Furthermore, in both of these models, the combined inhibition of both VEGFR and EGFR pathways with bevacizumab and erlotinib, or the dual VEGFR/EGFR inhibitor vandetanib, reduced pericyte coverage and extended progression-free survival (13). These findings support the hypothesis that EGFR signaling in pericytes is implicated in the resistance to VEGF/R blockade in NSCLC and suggest that dual targeting may delay the emergence of resistance.

The authors' findings are in line with published data suggesting that pericytes might confer resistance or refractoriness to antiVEGF/R therapies. Dual pharmacological targeting of both VEGFR and PDGFR signaling in endothelial cells and pericytes has been shown to be more effective than inhibition of VEGFR alone (14), and mature pericyte-covered vessels show relative resistance to pruning when treated with VEGFR inhibitors (15). In sharp contrast, Nisancioglu et al. used the pericyte-deficient pdgf- $\mathrm{b}^{\text {ret } / \text { ret }}$ mouse to show there is no effect of VEGF inhibition on tumor growth or vascular density in pericyte-deficient tumors compared with controls (16). Overall, these data emphasize the interdependence of pericytes and endothelial cells in tumors and the importance of tumor phenotype in determining the effects of targeting endothelial cells and/or pericytes in tumor vessels $(17,18)$.

Are there other stromal cells playing a role in acquired resistance to antiangiogenics? Cascone et al. show that p-EGFR increases in perivascular cells, but there is also substantial p-EGFR immunostaining far from vessels (13). This observation - together with the finding of differential localization of $\alpha$-SMA-positive cells (fibroblasts and myofibroblasts) in resistant tumors, even though total abundance is not changed - suggests that there are changes in other cell components in the resistant tumors, some of which could be mesenchymal cells or fibroblasts. Thus, these findings are indicative of broader stromal remodeling with adaptation of several different cellular components.

\section{Context-dependent stromal} adaptation to induce resistance

Evidence from different experimental settings suggests that multiple mechanisms of resistance may arise depending on the tumor type and microenvironment, and these context-dependent changes in the stroma are critical for resistance to antiangiogenic therapies. For instance, whereas refractoriness to therapy has been associated in some cases to the presence of bone marrow-derived $\mathrm{Gr}-1^{+} \mathrm{CD} 11 \mathrm{~b}^{+}$myeloid suppressor (i.e., dendritic) cells $(11,12)$, Cascone et al. illustrate two adaptive resistance settings in which tumor-associated macrophage recruitment is not altered upon therapy (13), further confirming some mechanistic differences between intrinsic and acquired resistance models. Furthermore, the two acquired resistance models show differences in their stromal adaptation, with upregulation of FGFR signaling in the subcutaneous stromalpoor model (H1975 cells) but not in the orthotopic stromal-rich model (H441 cells); these results indicate that the tumor microenvironment plays a key role in specific adaptation changes for resistance.

A possible explanation for these diverse adaptation mechanisms could be related to the repertoire of proangiogenic growth factors expressed in each tumor type. Indeed, several ligands of the EGF family have been identified as limiting mediators of angiogenic switching and neovascularization with proangiogenic signaling through EGFR in pericytes (19). Furthermore, tumor phenotype, determined as the range and compartmentalization of ligands expressed, is key in determining the antitumor effectiveness of inhibiting endothelial cells or pericytes (17). Thus, it is plausible that the repertoire of proangiogenic growth factors expressed in each tumor setting are driving the type of stromal adaptation that could sometimes implicate EGFR in endothelial cells, EGFR in pericytes, or PDGFR in pericytes, depending on the tumor context.

\section{Clinical relevance}

Cascone et al. show in two acquired resistance models that the combined inhibition of both VEGFR and EGFR pathways extends progression-free survival compared with monotherapy (13). These findings not only demonstrate that targeting such pathways improves therapeutic efficacy, but also strengthen the postulate that dual VEGFR/ EGFR targeting delays the emergence of resistance. Therefore, these preclinical results may lead one to infer that dual inhibition of VEGF/R concomitant with EGFR pathways in NSCLC patients would trans- late into better and longer-lasting efficacy by impeding this resistance mechanism. Indeed, a phase IIIb clinical trial, ATLAS, compared single VEGF inhibition using bevacizumab with double VEGFR/EGFR inhibition by adding erlotinib in previously treated NSCLC patients, and the combination therapy demonstrated a significant improvement in progression-free and overall survival $(20,21)$. Thus, these significant but modest improvements in outcome with dual VEGFR/EGFR inhibition in NSCLC suggest that targeting multiple stromal resistance pathways may delay the onset of therapeutic resistance.

\section{Acknowledgments}

The author would like to thank Francesc Viñals and Mariona Graupera for critical reading of the manuscript and helpful suggestions. The author is a "Ramon y Cajal" fellow from MICINN, and his work is supported by research grants from MICINN (SAF2009-08375 and RTICC-RD20060092) and AGAUR (SGR727) from Spain.

Address correspondence to: Oriol Casanovas, Tumor Angiogenesis Group, Translational Research Laboratory, Catalan Institute of Oncology - IDIBELL, Av Gran Via de l'Hospitalet, 199-203. (3a pl.), E-08907 L'Hospitalet de Llobregat, Spain. Phone: 34.93.260.7344; Fax: 34.93.260.7466; E-mail: ocasanovas@iconcologia.net.

1. Folkman J. Angiogenesis: an organizing principle for drug discovery? Nat Rev Drug Discov. 2007; 6(4):273-286

2. Boehm T, Folkman J, Browder T, O'Reilly MS. Antiangiogenic therapy of experimental cancer does not induce acquired drug resistance. Nature. 1997; 390(6658):404-407.

3. Rini BI, Atkins MB. Resistance to targeted therapy in renal-cell carcinoma. Lancet Oncol. 2009; 10(10):992-1000.

4. Ellis LM, Hicklin DJ. Pathways mediating resistance to vascular endothelial growth factor-targeted therapy. Clin Cancer Res. 2008;14(20):6371-6375.

5. Kerbel RS, et al. Possible mechanisms of acquired resistance to anti-angiogenic drugs: implications for the use of combination therapy approaches. Cancer Metastasis Rev. 2001;20(1-2):79-86.

6. CarmelietP. Angiogenesis in life, disease and medicine. Nature. 2005;438(7070):932-936.

7. Bergers G, Hanahan D. Modes of resistance to anti-angiogenic therapy. Nat Rev Cancer. 2008; 8(8):592-603.

8. Crawford Y, Ferrara N. Tumor and stromal pathways mediating refractoriness/resistance to antiangiogenic therapies. Trends Pharmacol Sci. 2009; 30(12):624-630.

9. Ebos JM, Lee CR, Kerbel RS. Tumor and host-mediated pathways of resistance and disease progression in response to antiangiogenic therapy. Clin Cancer Res. 2009;15(16):5020-5025.

10. Crawford Y, et al. PDGF-C mediates the angiogenic and tumorigenic properties of fibroblasts associated with tumors refractory to anti-VEGF treatment. 
Cancer Cell. 2009;15(1):21-34.

11. Shojaei $F$, et al. Tumor refractoriness to anti-VEGF treatment is mediated by CD $11 \mathrm{~b}+\mathrm{Gr} 1+$ myeloid cells. Nat Biotechnol. 2007;25(8):911-920.

12. Shojaei F, et al. G-CSF-initiated myeloid cell mobilization and angiogenesis mediate tumor refractoriness to anti-VEGF therapy in mouse models. Proc Natl Acad Sci U S A. 2009;106(16):6742-6747.

13. Cascone $T$, et al. Upregulated stromal EGFR and vascular remodeling in mouse xenograft models of angiogenesis inhibitor-resistant human lung adenocarcinoma. J Clin Invest. 2011;121(4):1313-1328.

14. Bergers G, Song S, Meyer-Morse N, Bergsland E, Hanahan D. Benefits of targeting both pericytes and endothelial cells in the tumor vasculature with kinase inhibitors. J Clin Invest. 2003;111(9):1287-1295.

15. Helfrich I, et al. Resistance to antiangiogenic ther- apy is directed by vascular phenotype, vessel stabilization, and maturation in malignant melanoma. J Exp Med. 2010;207(3):491-503.

16. Nisancioglu MH, Betsholtz C, Genove G. The absence of pericytes does not increase the sensitivity of tumor vasculature to vascular endothelial growth factor-A blockade. Cancer Res. 2010;70(12):5109-5115.

17. Sennino B, et al. Cellular source and amount of vascular endothelial growth factor and platelet-derived growth factor in tumors determine response to angiogenesis inhibitors. Cancer Res. 2009; 69(10):4527-4536.

18. Lu C, et al. Targeting pericytes with a PDGF-B aptamer in human ovarian carcinoma models. Cancer Biol Ther. 2010;9(3):176-182.

19. Nolan-Stevaux O, et al. Differential contribution to neuroendocrine tumorigenesis of parallel Egfr signaling in cancer cells and pericytes. Genes Cancer. 2010;1(2):125-141.

20. Miller VA, O'Connor P, Soh C, Kabbinavar F. A randomized, double-blind, placebo-controlled, phase IIIb trial (ATLAS) comparing bevacizumab (B) therapy with or without erlotinib (E) after completion of chemotherapy with B for first-line treatment of locally advanced recurrent, or metastatic non-small cell lung cancer (NSCLC). J Clin Oncol. 2009;27(suppl):S18.

21. Kabbinavar FF, et al. Overall survival (OS) in ATLAS, a phase IIIb trial comparing bevacizumab (B) therapy with or without erlotinib (E) after completion of chemotherapy (chemo) with B for first-line treatment of locally advanced, recurrent, or metastatic non-small cell lung cancer (NSCLC). JClin Oncol. 2010;28(suppl):Abstract 7526.

\section{Tolerogenic pDCs: spotlight on Foxo3}

\section{Vincenzo Bronte}

Verona University Hospital and Department of Pathology, Immunology Section, University of Verona, Verona, Italy.

\begin{abstract}
Cancer creates a peculiar inflammatory environment enriched for transcription factors with a negative influence on adaptive immunity. In this issue of the JCI, Watkins and colleagues identify Foxo3 as a master regulator of the tolerogenic program in tumor-associated, plasmacytoid DCs (pDCs). Foxo3 enables pDCs to induce tolerance in tumor antigen-specific $\mathrm{CD8}^{+} \mathrm{T}$ cells, turning them into regulatory lymphocytes capable of inhibiting nearby $\mathrm{CD8}^{+} \mathrm{T}$ lymphocytes. Provision of tumor-specific $\mathrm{CD}^{+}$ $T$ helper cells interrupts this circuit by inhibiting Foxo3 expression and fully licensing the antigen-presenting ability of pDCs. These data identify a new target for therapeutic intervention and provide insight into the transcription factor interplay in myeloid cells recruited to the cancer microenvironment.
\end{abstract}

Complex responses to inflammatory stimuli require a sophisticated and coordinated transcriptional regulation activated by dedicated transcription factors (TFs) both at the single cell and tissue level. After LPS stimulation, a classical stimulus of the innate immune system, macrophages activate at least three classes of TFs: class I TFs that are shared by various cells and activated by signal-regulated, posttranslational modification; class II TFs that are synthesized de novo after stimulation; and class III TFs that are induced during cell differentiation and control complex phenotypic and functional changes (1). Studies in cancer are unveiling a network of negative transcriptional regulators of adaptive immunity in myeloid cells, which includes various members potentially belonging to the class III family.

Conflict of interest: The author has declared that no conflict of interest exists.

Citation for this article: J Clin Invest. doi:10.1172/ JCI57190.
The work from Watkins and colleagues reported in this issue of JCI identifies forkhead box O3 (Foxo3) as the main regulator of the immunosuppressive program in tumor-associated DCs (TADCs), which are mainly plasmacytoid DCs (pDCs), infiltrating prostate cancers (2). Human $\mathrm{CD} 123^{+} / \mathrm{CD} 304^{+} / \mathrm{CD} 11 \mathrm{c}^{-}$and mouse

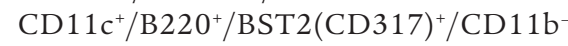
pDCs were isolated from either human prostate cancer specimens or cancers arising in transgenic adenocarcinoma of the mouse prostate (TRAMP) mice. Tumorconditioned pDCs but not pDCs isolated from other anatomical districts of tumorfree mice (including normal prostate) induced unresponsiveness to second stimulation with the antigen - that is, bona fide tolerance - in $\mathrm{CD}^{+} \mathrm{T}$ cells. Tolerance was antigen-specific and "infectious," since the $\mathrm{pDC}$-tolerized CD8 ${ }^{+} \mathrm{T}$ cells prevented naive $T$ cell proliferation in an antigen-unspecific manner, acting like Treg lymphocytes (Figure 1). Although pDCs from mouse tumor-draining lymph nodes were previously shown to directly activate mature $\mathrm{CD}^{+}$Tregs and prevent their conversion to $T$ helper 17 cells through a pathway requiring the activity of the enzyme indoleamine 2,3-dioxygenase (IDO) $(3,4)$, this work shows for the first time a direct conversion of $\mathrm{CD}^{+} \mathrm{T}$ cells toward a regulatory program.

\section{TADCs convert CD8+ $\mathrm{T}$ cells}

Watkins et al. found that TADC depletion by injection of anti-CD317 antibody resulted in increased activation and reduced Treg function of adoptively transferred $\mathrm{CD}^{+} \mathrm{T}$ cells reaching tumor mass: these cytotoxic $\mathrm{T}$ cells were specific for the main tumor antigen, the oncogene SV40, which drives tumorigenesis in TRAMP mice. TADC elimination also resulted in therapeutic benefit, since total urogenital tract and prostate weights, both indicators of tumor burden, were reduced in TADC-depleted TRAMP mice compared with those in control mice. Surprisingly, two amino acid metabolizing enzymes were upregulated in the TADCs: IDO and arginase 1 (ARG1). The authors used chemical inhibitors to provide evidence that these enzymes might be involved in the suppressive activity of TADCs on $\mathrm{CD}^{+} \mathrm{T}$ cells. However, only IDO inhibitors were effective in vitro, whereas both IDO and ARG inhibitors temporarily restored immune reactivity in vivo, indicating that ARG inhibitors might target this enzyme in other tumor-infiltrating cells, such as macrophages or subsets of classic CD $11 b^{+} / \mathrm{CD} 11 \mathrm{c}^{\text {hi }} / \mathrm{MHC} \mathrm{II}^{+}$ DCs, whose ARG1 overexpression was 